\title{
MATTER EFFECTS IN NEUTRINO PROPAGATION
}

\author{
A. YU. SMIRNOV \\ Institute for Nuclear Research \\ Academy of Sciences of the USSR \\ 60th October Anniversary prospect 7a \\ 117312 Moscou \\ USSR
}

\begin{abstract}
Conditions and dynamics of the resonant neutrino conversion are described. We discuss the applications of the effect to the solar neutrinos as well as present status of the conversion inside the Sun. The influence of different matter density perturbations on the conversion is considered, and in this connection the possible effects of parametric and stochastic enhancement of the influence are remarked.
\end{abstract}

\section{Introduction}

Resonant neutrino conversion is the process of nonreversible transformation of one neutrino species into another one (in a system of mixed neutrinos) during the propagation through the matter with monotonously changing density. The transformation occurs continuously according to density change, and mainly, in the resonance layer (the layer in which the density varies in definite limits about the resonance value) $[1,2]$.

According to definition the key points are : 1) mixing of neutrinos - the transitions occur between mixed neutrino components; 2) interactions of neutrinos with matter - the elastic forward scattering is essential [3]; 3) resonance (crossing of the resonance layer); 4) change of density, moreover the density should change slowly to satisfy the adiabaticity condition. In the first part of review we consider these items and then describe dynamics of conversion (for more details see reviews [4-6]).

Conditions of resonance conversion are fulfilled for the Sun in a wide region of neutrino parameters (mass difference $\Delta m^{2}=m_{2}^{2}-m_{1}^{2}$ and mixing angle, $\theta)$ [1]. The conversion $\left(v_{\mathrm{e}}-v_{\mu}, v_{\mathrm{e}}-v_{\tau}\right)$ diminishes signals in the solar neutrino experiments $: v_{\mu}$ or $v_{\tau}$ are sterile at the low energies with respect to charged currents interactions. The effect depends on neutrino energy, and consequently the conversion distorts the energy 
spectrum of the electron neutrinos. Conversion may solve the solar neutrino problem [1]. It can ensure up to 15 -fold suppression of the argon production rate in $\mathrm{C} 1$ - Ar -experiment, and rather independent suppression of $v$ e-scattering signal (Kamiokande-II). In a view of existing data and forthcoming $\mathrm{Ga}$ - Ge -experiment - results the present status of resonant conversion will be discussed.

In the third part of the review we consider the influence of different matter density perturbations on resonant conversion. Such a consideration has two aspects. Firstly, it enables to understand the sensitivity of the conversion effects to the model of the Sun and to evaluate the possibility of measuring the solar parameters with neutrino data. Secondly, if the perturbations depend on time then variations of $v_{e}$ -flux are induced via the resonant conversion. The parametric and stochastic enhancement of perturbation effects will be remarked.

\section{Resonant neutrino conversion}

\subsection{CONDITIONS OF RESONANT CONVERSION}

2.1.1. Mixing. The necessary condition of resonant conversion in a system of two neutrinos, for example $v_{e}-v_{\mu}$, is the mixing of these neutrinos. Mixing implies the interaction, which transforms $v_{e}$ into $v_{\mu}$. In the simplest case the mixing (so called vacuum mixing) is induced by the nondiagonal mass terms of the Hamiltonian. Now $v_{e}$ and $v_{\mu}$ which are called the flavor states or eigenstates of the weak interactions turn out to be the coherent mixtures of $v_{1}$ and $v_{2}$-- the states with definite masses $m_{1}$ and $m_{2}$ (eigenstates of the mass matrix):

$$
\begin{aligned}
& v_{e}=\cos \theta v_{1}+\sin \theta v_{2} \\
& v_{\mu}=-\sin \theta v_{1}+\cos \theta v_{2}
\end{aligned}
$$

Here $\theta$ is the mixing angle. Flavor states themselves have no definite masses.

2.1.2. Refraction. Conversion is stipulated by the interactions of neutrinos with matter, and precisely -- by the elastic forward scattering, which is reduced to the appearance of the refraction indexes, $n_{e}$ and $n_{\mu}$, for neutrino waves [3] :

$$
(n-1) \propto G_{F} N / k
$$

Here $G_{F}$ is the Fermi constant, $N$ is the concentration of particles in medium, $k$ is the momentum of neutrino. Matter influences on the evolution of mixed neutrinos if the indexes are different $n_{e} \neq n_{\mu}$. So, the conversion takes place in the transparent for neutrinos mediums, 
being nonsymetric with respect to the mixed components. The influence is described by the refraction length, $1_{0}$, defined as the distance over which an additional phase difference between $v_{e}$ an $v_{\mu}$ waves due to interactions becomes $2 \pi$ [3]. The $l_{0}$ determines spatial scale of matter effects. For $v_{e}-v_{\mu}$-system one has

$$
l_{0}=\frac{2 \pi}{k\left|n_{e}-n_{\mu}\right|}=\frac{2 \pi}{\sqrt{2} G_{F} N_{e}}=\frac{2 \pi m_{N}}{\sqrt{2} G_{F} \rho^{e f f}}
$$

where $N_{e}=\rho . Y_{e} / m_{N}$ is the electron concentration, $\rho$ eff $=\rho . Y_{e}$ is the effective density, $\mathrm{m}_{\mathrm{N}}$ is the nucleon mass. The corresponding width of matter :

$$
d_{0}=l_{0} \bullet \rho=\frac{2 \pi m_{N}}{\sqrt{2} G_{F}}=3.510^{9} \mathrm{~g} / \mathrm{cm}^{2}
$$

is the universal constant, which in fact fixes the field of applications of the phenomena : the width $d \geq d_{0}$ is needed.

Matter effects can be described equivalently in terms of potentials, $V_{e}$ and $V_{\mu}$, in which $v_{\mathrm{e}}$ and $v_{\mu}$ are moving: $V \propto(n-1) k \propto G_{F} N_{[7]}$

2.1.3. Neutrino eigenstates in matter. Effective mixing in matter. Effective (dynamical) mixing is used for the description of neutrino evolution [3]. In matter this mixing is introduced with respect to the neutrino eigenstates in matter, $v_{1 \mathrm{~m}}$ and $v_{2 \mathrm{~m}}$, which are determined as the eigenstates of the total Hamiltonian, including the neutrino interactions (i.e. potentials $V_{e}$ and $V_{\mu}$ ):

$$
\begin{aligned}
& v_{e}=\cos \theta_{m} v_{1 m}+\sin \theta_{m} v_{2 m} \\
& v_{\mu}=-\sin \theta_{m} v_{1 m}+\cos \theta_{m} v_{2 m}
\end{aligned}
$$

$\theta_{m}$ is the mixing angle in matter. The eigenstates $v_{i m}$ have definite energies (eigenvalues) as well as definite phase and group velocities. In vacuum $v_{i m}$ coincide with $v_{i}$ and $\theta_{m}=\theta$. Matter changes mixing, and moreover $\theta_{\mathrm{m}}$ depends on matter density. As it can be shown at $\rho^{\text {eff }} \neq 0$ the transitions $v_{1} \div v_{2}$ takes place and hence $v_{i}$ are no more the eigenstates of the Hamiltonian : $v_{i m} \neq v_{i}$. 


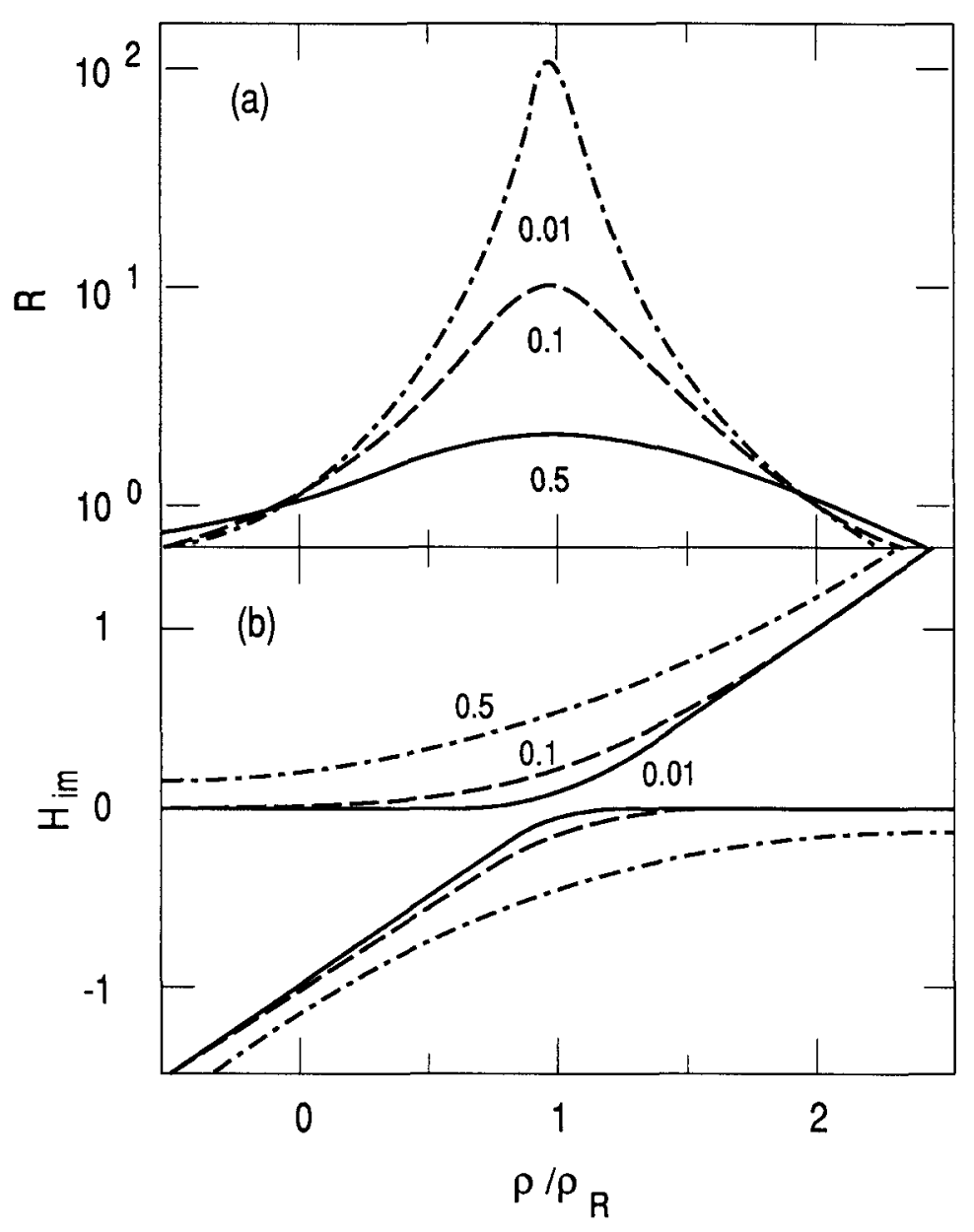

Figure 1. The dependence of (a) the resonance factor $R=\sin ^{2} 2 \theta_{m} / \sin ^{2} 2 \theta$ and $(b)$ level energies in units $\Delta m^{2} \cdot(2 k \cos 2 \theta)^{-1}$ on the effective density for different value of $\sin ^{2} 2 \theta$ (numbers of the curves).

2.1.4. Resonance. The dependance of mixing parameter, $\sin ^{2} 2 \theta_{\mathrm{m}}$, on the density has a resonance character [1] (fig.1). At

$$
\rho_{R}=\frac{m_{N} \cos 2 \theta}{2 \sqrt{2} G_{F}} \cdot \frac{\Delta m^{2}}{E}
$$

where $\Delta m^{2}=m_{2}^{2}-m_{1}^{2}$ and $\mathrm{E}$ is the energy of neutrino, this parameter reaches the maximum $: \sin ^{2} 2 \theta_{m}=1$ for arbitrary small $\theta$. The $\rho_{\mathrm{R}}$ is called the resonant density. Half width of resonance at half height is proportional to the vacuum mixing : 


$$
\Delta \rho_{R}=\rho_{R} \cdot \tan 2 \theta
$$

The width of resonance layer, $2 \Delta \rho_{R}$ fixes the scale on which resonant conversion occurs.

Parameter $\sin ^{2} 2 \theta_{m}$ defines (as $\sin ^{2} 2 \theta$ does in vacuum) the depth of neutrino oscillations. Therefore in matter with resonant density the depth is maximal.

Resonance condition (4) can be written as

$$
l_{\nu}=l_{0^{*}} \cos 2 \theta
$$

where $\mathrm{l}_{V}=4 \pi \mathrm{E} / \Delta \mathrm{m}^{2}$ is the vacuum oscillation length. According to (6) in resonance (at small $\theta$ ) period of system $\left(l_{\nu}\right)$ itself coincides with period of external medium $\left(l_{0}\right)$.

The value $\rho=\rho_{R}$ is the specific point in the dependance of $\theta_{m}$ on $\rho$. When $\rho$ varies from $\rho \gg \rho_{R}$ to zero, the angle $\theta_{m}$ diminishes from $\pi / 2$ to $\theta$. At $\rho=\rho_{R}$ the $\theta_{m}=\pi / 4$.

2.1.5. Flavor changing of neutrino eigenstates. Mixing angle $\theta_{\mathrm{m}}$ determines according to (3) a flavor, or $v_{e^{-}}, v_{\mu}$-content, of the neutrino eigenstates, $v_{\mathrm{im}}$. Propagating through a matter with varying density the $v_{i m}$ change their flavors. If $\theta$ is small and $\rho$ varies from $\rho \gg \rho_{R}$ to $\rho$ « $\rho_{R}$, the flavors of $v_{i m}$ change almost completely. For example, $v_{2 m}$ coincides practically with $v_{\mathrm{e}}$ at $\rho \geqslant \rho_{\mathrm{R}}$ and with $v_{\mu}$ at $\rho \ll \rho_{\mathrm{R}}$. Mainly a flavor changes in the resonance layer: $\rho_{R}-\Delta \rho_{R}<\rho<\rho_{R}+\Delta \rho_{R}$.

2.1.6 Level crossing. Cabibbo [8] and Bethe [7] had given the interpretation of the resonance in terms of eigenvalues $\mathrm{H}_{\mathrm{im}}$ i.e. the level energies corresponding to $v_{i m}$ (fig. $1 b$ ). Potentials, $V_{e}$ and $V_{\mu}$, are proportional to a density, and consequently the total energies of $v_{e}$ and $v_{\mu}, H_{e}$ and $H_{\mu}$, are the linear functions of $\rho$. At resonance they cross :

$$
H_{e}\left(\rho_{R}\right)=H_{\mu}\left(\rho_{R}\right)
$$

Mixing of $v_{e}$ and $v_{\mu}$ rejects level crossing : the eigenvalues $H_{i m}(\rho)$, are not equal but their splitting is minimal in resonance (fig. $1 \mathrm{~b}$ ). In the resonance layer when $\rho$ increases the curve $\mathrm{H}_{1 \mathrm{~m}}(\rho)$ goes from the line $H_{e}(\rho)$ to the line $H_{\mu}(\rho)$, the $H_{2 m}(\rho)$ vice versa -- from $H_{\mu}(\rho)$ to $H_{e}(\rho)$.

In resonance the difference of the potentials $V=V_{\mu}-V_{e}$, compensates the difference of level energies related to masses. As the $\mathrm{V}$ for neutrinos and antineutrinos have an opposite sign the resonance as 
well as resonant conversion in a given medium takes place for neutrinos or antineutrinos only, depending on the respective signs of $\Delta \mathrm{m}^{2}, V$ and $\cos 2 \theta$.

2.1.7. Adiabaticity. $[1,2,9,10]$. Adiabaticity implies a slowness of density change. If $\rho$ changes adiabatically (slowly), then the transitions between the eigenstates $v_{1 m}-v_{2} m$ can be neglected; the admixtures of $v_{i m}$ in a given $v$-state conserve; the system has a time to adjust itself to external condition (density) variations. The condition of adiabaticity can be written as

$$
\frac{d \theta_{m}}{d r} \ll \frac{d \varphi}{d r} \equiv\left|H_{2 m}-H_{1 m}\right|
$$

where $\varphi$ is the phase difference between $v_{1 \mathrm{~m}}$ and $v_{2 \mathrm{~m}}$ waves $\left(d \theta_{m}(\rho) / d r \propto d \rho / d r\right)$. The condition is the most crucial in resonance, where the level splitting is minimal $[1,2]$ :

$$
2 \Delta r_{R} \geq l_{m}^{R}
$$

Here $\Delta r_{R}=10 \cdot \tan 2 \theta,\left[1_{\rho} \equiv(d \rho / d r)^{-1} \cdot \rho\right]$ is the spatial half width of the resonance layer; $l_{m}^{R}$ is the oscillation length in resonance : $l_{m}^{R}=l_{\text {w }} \sin 2 \theta$. According to (9) at least one oscillation length should be obtained in resonance layer.

The degree of adiabaticity violation is determined by the adiabaticity parameter [4] :

$$
\mathfrak{x}_{R}=\frac{\Delta r_{R}}{l_{m}^{R}}=\frac{\sin ^{2} 2 \theta}{4 \pi \cos 2 \theta} \cdot \frac{\Delta m^{2}}{E} \cdot l_{\rho}
$$

At $\mathfrak{x}_{R} \leq 0.1$ the violation is strong and the transitions $v_{1 \mathrm{~m}} \rightarrow v_{2 \mathrm{~m}}$ become essential.

So far we have considered main conditions of resonance conversion : 1) mixing, 2) resonance (level crossing), 3) adiabaticity (see also the general discussion in [1]).

\subsection{DYNAMICS OF RESONANT CONVERSION.}

Resonant neutrino conversion is, in fact, the change of the flavor of neutrino state on the adiabatic (or weakly nonadiabatic) crossing of resonance layer. The decomposition of given $v$-state over $v$ eigenstates, $v_{i m}$, and the change the flavors of $v_{i m}$ themselves are all we need to trace the conversion. The dynamics is reduced to the change of the admixtures of $v_{i m}$ in $v(t)$. 
To describe the process of conversion the survival probability, $P(t)$, i.e. the probability to find the neutrino of initial type in a given moment $t$, is used.

One can single out three different regimes of conversion 1) nonoscillatory, 2) oscillatory adiabatic, and 3) nonadiabatic.

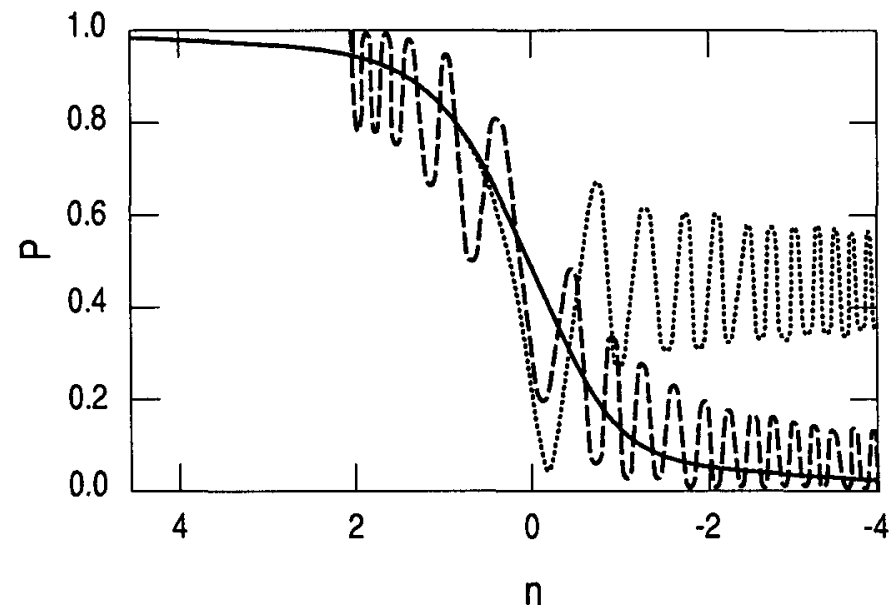

Figure 2. Spatial picture of resonant neutrino conversion. The dependence of survival probability on the distance from the resonance layer : $n=\left(\rho-\rho_{R}\right) / \Delta \rho_{R}$ for nonoscillatory transformation (solid line), oscillatory adiabatic transition (dashed line), adiabaticity violation regime (dotted line).

2.2.1. Nonoscillatory transition $[1,2]$. Let neutrinos, for definiteness $v_{e}$, are produced at $\rho \geqslant \rho_{R}$ [ more precisely at $\left.\left(\rho-\rho_{R}\right) / \Delta \rho_{R} » 1\right]$, and the adiabaticity is satisfied during the propagation. Then in the initial moment according to the first condition $\left(\rho \gg \rho_{R}\right)$ the mixing angle equals $\pi / 2$ and hence the neutrino state coincides practically with $v_{2 m}$ : $v\left(t_{0}\right)=v_{e} \cong v_{2 m}$. According to the second condition (adiabaticity) $v(t)$ will coincide with $v_{2} m$ throughout the propagation. Consequently, $v(t)$ changes its flavor together with $v_{2 m}$; the flavor of $v(t)$ follows density variations, like the flavor of $v_{2} \mathrm{~m}$ does (fig. 2). If the final density is zero (or « $\rho_{R}$ ), then at the exit one has $v\left(t_{f}\right)=v_{2 m}(\rho=0)=v_{2}=v_{e} \sin \theta+v_{\mu} \cos \theta$ and then the survival probability is [1] :

$$
P=P\left(v_{e} \rightarrow v_{e}\right)=\sin ^{2} \theta
$$

The smaller vacuum mixing, the stronger transformation of the neutrino type into another one can be achieved in contrast with vacuum oscillations. 
2.2.2. Adiabatic oscillatory transition [2]. let the adiabaticity condition is fulfilled but the neutrinos are produced not far from thr resonance layer $:\left|\rho_{0}-\rho_{R}\right|<$ several $\Delta \rho_{R}$. Then $v(t)$ contains the comparable admixtures of both eigenstates and moreover these admixtures will conserve. As $v(t)$ does not coincide with one of the eigenstates the flavor of $v(t)$ oscillates. Oscillations superpose on the conversion (fig.2), but the averaged probability changes according to density variations $[2,9,10]$ :

$$
\bar{P}=\frac{1}{2}-\frac{1}{2} \cos 2 \theta_{m}\left(\rho_{0}\right) \cos 2 \theta_{m}(\rho)
$$

when $\rho_{0}$ approaches $\rho_{R}$, the transformation becomes weaker : $P$ increases. At $\rho_{0}=\rho_{R}: P=1 / 2$. (In the adiabatic regime not only $P$ but also the depth of oscillations, $A p=\sin 2 \theta_{m}\left(\rho_{0}\right) \cdot \sin 2 \theta_{m}(\rho)$ depends on the initial and final densities only and does not depend on density distribution in the intermediate points.

2.2.3. Adiabaticity violation regime. If the adiabaticity is violated, the admixtures of neutrino eigenstates in a given $v(t)$ change. Let neutrinos are produced far from the resonance layer $\left(\rho_{0} \geqslant \rho_{R}\right)$, then $v\left(t_{0}\right)=v_{e}$ consists of $v_{2} \mathrm{~m}$ predominatingly. But the admixture of $v_{1 \mathrm{~m}}$ appears in $v(t)$ in the course of propagation. Consequently the probability $P(t)$ begins to oscillate and the transition becomes weaker, than in nonoscillatory case (fig. 2 ). The averaged probability can be written now as

$$
\bar{P}=\frac{1}{2}+\left(\frac{1}{2}-P_{21}\right) \cos 2 \theta_{m}\left(\rho_{0}\right) \cos 2 \theta_{m}(\rho)
$$

where $\mathrm{P}_{21}$ is the probability of $v_{2 m}-v_{1 m}$ transition At small $\theta$ and at the values of $\rho_{0}$ and $\rho_{f}$ beyond the resonance layer, the $P_{21}$ can be evaluated using the well known Landau-Zener result $[12,13]$. It gives the probability of transition between two levels under linear with time perturbation, which mixes these levels and induces their crossing :

$$
P_{21}=\exp \left(-\pi^{2} \mathfrak{x}_{R}\right)
$$

There is a lot of improvements and generalizations of formula (14) in literature [14].

2.2.4. Analogies. There are many analogies of resonant conversion in different fields of physics. Let remark two of them.

The system of mixed neutrinos is similar to the weakly coupled oscillators (for example, two pendulums) [15]. Matter effect is 
equivalent to a change of pendulum eigenfrequences $\omega_{1}$ and $\omega_{2}$. Resonance corresponds to the equality $\omega_{1}=\omega_{2}$. Now the conversion is nonreversible transmission of oscillations from one pendulum to another one under slow changing of frequences from $\omega_{1} \ll \omega_{2}$ to $\omega_{1}$ " $\omega_{2}$.

Another analogy is the electron spin flip in the rotating magnetic field [16]. The states with the projections $1 / 2$ and $-1 / 2$ correspond to $v_{e}$ and $v_{\mathbf{m}}$.

\subsection{DIFFERENT REALIZATIONS OF RESONANT NEUTRINO CONVERSION.}

The general conditions : mixing, level crossing, adiabaticity may have many realizations even for neutrino systems [11]. Depending on properties of neutrino states are mixed one can single out three types of conversion.

2.3.1. Flavor conversion (considered above). Neutrino states with different flavors but the same helicities are involved, for example $n_{\mathrm{eL}}$ -

$\mathrm{n}_{\mu \mathrm{L}}$. In the course of conversion the flavor changes, and the helicity conserves. In general all three known neutrino species, $v_{e}, v_{\mu}, v_{t}$, are mixed. Such a system has three resonances but in usual medium (electrons, nuclei) in the lowest order of the perturbation theory the $v_{\mu}$ and $v_{\tau}$ interact equally and only two level crossings are possible. In $\left(v_{\mathrm{e}}-v_{\mu}\right)$ and $\left(v_{\mathrm{e}}-v_{\tau}\right)$-chanels [17]. If $\Delta m_{21}^{2}=m_{2}^{2}-m_{1}^{2}$ differs from $\Delta m_{31}^{2}=m_{3}^{2}-m_{1}^{2}$ sufficiently (which realizes for mass hierarchy, $\mathrm{m}_{1}$ « $\mathrm{m}_{2}$ « $\left.\mathrm{m}_{3}\right)$, then the corresponding resonances are splitted on the density scale. In this case crossing of resonances can be described independently -- three neutrino task is reduced to two neutrino task (see reviews [4-6].

2.3.2.Spin conversion [18]. Conversion takes place in a system of left and right components composed the same dirac neutrino : $v_{\mathrm{eL}}$ and $v_{\mathrm{eR}}$. Mixing is induced by the interaction of neutrino magnetic moment, $\mu_{v}$, with magnetic field, B [the exchange (mixing) energy is $2 \mu_{v} \mathrm{~B}$ ]. Both level splitting and level crossing are stipulated by the refraction in inhomogenious matter. Now helicity is changed and flavor conserves.

2.3.3. Spin-flavor conversion. Both flavor and helicity of neutrino state are changed, for example $v_{e L}-v_{\mu R}[19]$. Mixing is induced by the interaction of the nondiagonal (transition) magnetic moment, $\mu_{\nu}$, with magnetic field. Now the mixing parameter is

$$
" \sin 2 \theta^{\prime \prime}=\left(2 \mu_{\vee} B\right) \cdot E / \Delta m^{2}
$$

Mass difference and refraction (potentials) give the contributions to level splitting. Level crossing is related to the change of density. 
Considered conversion types differ by the dependences of the adiabaticity parameter, $\mathfrak{x}_{R}$, on the neutrino energy. One has $\mathfrak{x}_{\mathbf{R}} \sim 1 / \mathrm{E}$ for the flavor conversion $(10), \mathfrak{x}_{R}=$ constant for the spin precession and

$$
\mathfrak{x}_{R}=\left(2 \mu_{v} B\right)^{2} \cdot E\left(\pi \Delta m^{2} l_{\rho}\right)
$$

for the spin flavor conversion. In the last case at $B=$ const, for example, $\mathfrak{x}_{R}$ is proportional to $\mathrm{E}$.

\section{Resonant conversion of solar neutrinos. Present status}

\subsection{EFFECTS OF FLAVOR CONVERSION}

Neutrinos, $v_{e}$, produced in the center of Sun, cross the layer of matter with monotonously decreasing density. Resonant flavor conversion results in suppression of the initial $v_{e}-f l u x$, which depends on $\Delta \mathrm{m}^{2}$, $\sin ^{2} 2 \theta$ and the energy of neutrinos :

$$
F\left(E, \Delta m^{2}, \sin ^{2} 2 \theta\right)=P\left(E, \Delta m^{2}, \sin ^{2} 2 \theta\right) \cdot F_{0}(E)
$$

Here $F_{0}$ is the flux without conversion. The suppression factor $p$ (averaged survival probability) as the function of $E / \Delta \mathrm{m}^{2}$ has a bath-like shape [1] (fig. 3).

Moreover there are two specific energies in $\mathrm{P}\left(\mathrm{E} / \Delta \mathrm{m}^{2}\right)$ dependence : $\mathrm{E}_{\mathrm{c}}$ and $\left.E_{a} .1\right)$. The energy of resonance turning on, $E_{c}$, is fixed via the resonance condition (4) by the central density $\rho_{c}$ :

$$
E_{d} / \Delta m^{2}=m_{N}\left(2 \sqrt{2} G_{F} \rho_{c}\right)
$$

For neutrinos with $\mathrm{E}<\mathrm{E}_{\mathrm{c}}$ there is no resonance (level) crossing. 2). The energy of adiabaticity violation, $E_{a}$, is determined by adiabaticity condition $\mathfrak{x}_{\mathrm{R}}\left(\mathrm{E}_{\mathrm{a}}\right)=0.5$ :

$$
E_{d} / \Delta m^{2}=l_{\rho} \cdot \sin 22 \theta(2 \pi \cos 2 \theta)
$$

(see $(9,10))$. When $\mathrm{E}$ increases the adiabaticity parameter diminishes : $\mathfrak{x}_{\mathrm{R}} \sim 1 / \mathrm{E}\left(l_{0}=\right.$ const. in a wide region of $\left.\rho\right)$, the adiabaticity violates stronger and $P$ increases $\left(E>E_{a}\right)$. The energy $E_{a}$ is proportional to $\sin ^{2} 2 \theta$.. with decreasing vacuum mixing the baths become narrower. 
If $E$ rises, the resonance layer shifts to the surface of the $S$ un. In the region of resonance turning on $\left(E=E_{c} \pm\right.$ several.$\left.\Delta E_{R}\right)$ the oscillatory adiabatic transition takes place, here $\Delta \mathrm{E}_{\mathrm{R}}=\mathrm{E}_{\mathrm{c}} \cdot \tan 2 \theta, \Delta \mathrm{E}_{\mathrm{R}}$ is the width of resonance in energy scale. At $\left(\mathrm{E}_{\mathrm{c}}+\right.$ several. $\left.\Delta \mathrm{E}_{\mathrm{R}}\right) \div \mathrm{E}_{\mathrm{a}}$ the conditions for the nonoscillatory transformation are fulfilled and $P=\sin ^{2} \theta$. At $E>$ $E_{a}$ neutrinos convert in adiabaticity violation regime (see sect.2.2.3., $(13,14)$. The mutual position of the suppression bath and the neutrino spectrum depends on $\Delta \mathrm{m}^{2}$, and if the spectrum falls on the edges of the bath, it distorts.

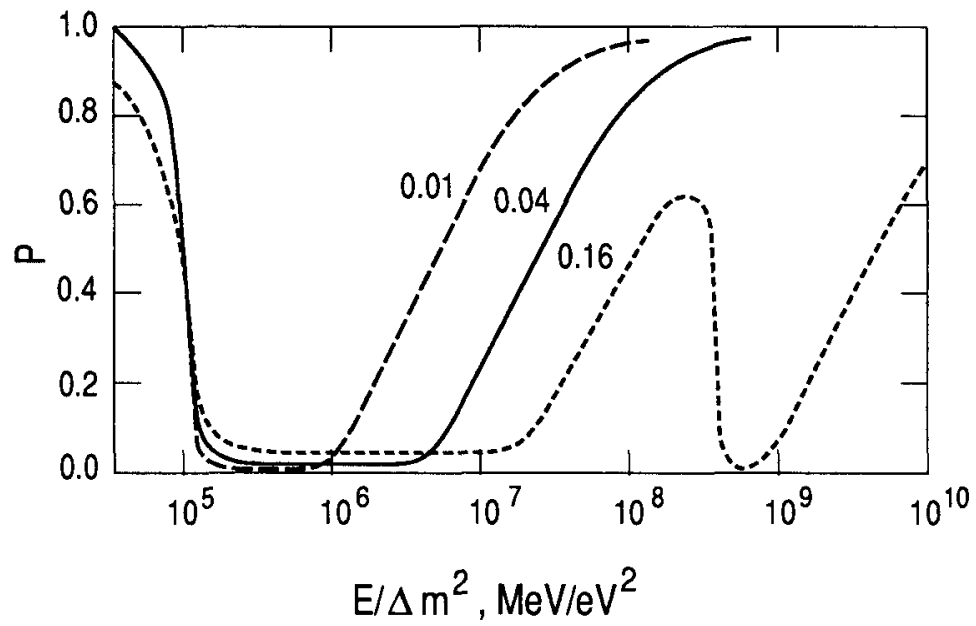

Figure 3. Suppression baths of flavor conversion. The dependences of suppression factors on $E / \Delta m^{2}$ for different values of $\sin ^{2} 2 \theta$ (numbers on the curves). The suppression pit for $3 v$-mixing (an example, dashed line).

Two remarks are in order.1). The averaging of $P$ over the $v$ production region results in the smoothing of the left edges of baths. 2) For three neutrino mixing the suppression factor $P(E)$ is the superposition of two baths, which are shifted on the axis $E$ one with respect to another by factor of $\Delta m_{13}^{2} / \Delta m_{12}^{2}$ (fig. 3).

\subsection{SPIN-FLAVOR CONVERSION AND SPIN CONVERSION}

In contrast with flavor conversion for spin flavor case [19] (1) mixing parameter $" \sin ^{2} 2 \theta$ is not constant : it depends on the magnetic field and the neutrino energy (15), (2) effective density is $\rho^{\text {eff }}=\rho\left(Y_{e}-Y_{n}\right)$ for $v_{e}-\bar{v}_{\mu}$, where $Y_{n}$ is the number of neutrons per nucleon, (3) the dependence of the adiabaticity parameter on the neutrino energy differs from that of flavor case. If $B(r) \sim \rho^{\alpha}$ and $\alpha<0.5$, then $\mathfrak{x}_{R}$ rises with increasing $E$ and the adiabaticity becomes better (or restores). This results in rather complicated shape of suppression pits, which in general 
differs from flavor conversion baths (especially on the bottom and nonadiabatic edge) fig.4 [20]. $P$ (E) depends on the magnetic field profile, which is essentially unknown. In some cases $(B(r))$ the shape of pit may coincide with that of the flavor conversion and the problem appears to distinguish these two cases.

For spin conversion the energy splitting is $\Delta H=G_{F}\left(\rho\left(Y_{e}-Y_{n} / 2\right) / m_{N}\right.$ and there is no level crossing inside the Sun. Moreover the effect does not depend on neutrino energy. It can be evaluated from the spin-flavor results by the transition $\Delta \mathrm{m}^{2} / \mathrm{E} \rightarrow 0$ ( $\mathrm{P}$ at the largest values of $\mathrm{E} / \Delta \mathrm{m}^{2}$ in fig. 4) [20].

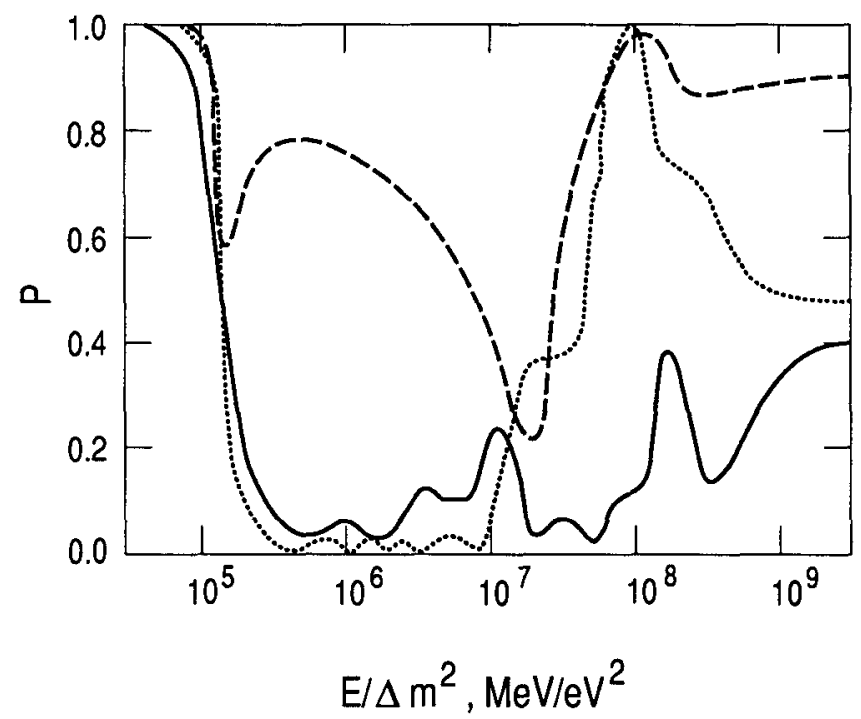

Figure 4. The suppression pits for spin-flavor conversion. The dependences of suppression factor on $E / \Delta m^{2}$ for different distributions of the magnetic field. Maximal strengh of field $B_{1}=10^{6}-10^{7}$ Gauss in the center of the $S$ un and $B_{o}=10^{4}$ Gauss in the convective zone were used (for more details see [20]. $\mu_{V} .=10^{-11} \mu B$.

\section{3 PRESENT STATUS OF RESONANT NEUTRINO CONVERSION}

The suppression factor for ${ }^{37} \mathrm{Ar}$-production rate in $\mathrm{Cl}$-Ar-experiment due to flavor conversion is

$$
R_{A r}\left(\Delta m^{2}, \sin ^{2} 2 \theta\right)=\frac{1}{Q_{A r}^{S S M}} \int d E d(E) F^{0}(E) P\left(E, \Delta m^{2}, \sin ^{2} 2 \theta\right)
$$

where $\mathrm{Q}^{S S M}{ }_{\mathrm{AR}}=\int \mathrm{dE} \sigma(\mathrm{E}) \mathrm{F}^{0}(\mathrm{E}), \sigma(\mathrm{E})$ is the cross-section, $\mathrm{F}^{\circ}(\mathrm{E})$ is the neutrino flux on the Earth in the standard solar model (SSM) [21]. The equation $R_{A r}\left(\Delta m^{2}, \sin ^{2} 2 \theta\right)=c=$ const determines lines of ${ }_{2} f$ equal 
suppression [1,2,4-6] (or ISOSNU lines [22] on $\Delta \mathrm{m}^{2}-\sin ^{2} 2 \theta$-plot

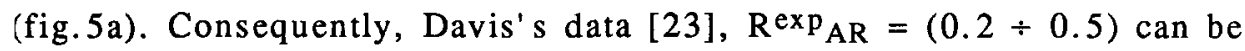
explaned in terms of resonant flavor conversion if the values of $\Delta \mathrm{m}^{2}$ and $\sin ^{2} 2 \theta$ find between ISOSNU lines $c_{\min }=R^{\exp } \min =0.2$ and $c_{\max }=0.5$. The system of three neutrinos is described by two points on $\Delta \mathrm{m}^{2}$ $\sin ^{2} 2 \theta$ - plot and as can be shown [24], to achieve the observed suppression of $\mathrm{QAr}_{\mathrm{Ar}}$ at least one point should be placed between ISOSNU lines $c_{\min }=R^{\exp } \min =0.2$ and $c_{\max }=\sqrt{R_{\max }^{\exp }}=0.7$.

The suppression of $v$ e-signal in Kamiokande-II experiment [25] which is sensitive to the high energy part of boron neutrino spectrum only, is rather independent on the suppression of $Q_{A r}$ (fig.5b) [24]. Preliminary Kamiokande-II result with $1 \sigma$ errors [25] excludes some part of loops in fig. 5 b. i.e. definite values of $\Delta \mathrm{m}^{2}$ and $\sin ^{2} 2 \theta$.

The suppression factor of Ge-production rate in $\mathrm{Ga}-\mathrm{Ge}$ experiment, $\mathrm{R}_{\mathrm{Ge}}$, is introduced similarly to (19). According to fig. 5c. at $\mathrm{R}_{\mathrm{Ar}}=0.2 \div$ 0.5 any result for $R_{G e}$ from 0.04 to 0.98 is possible and moreover $1 \sigma$ Kamiokande-II limit restricts this region of prediction very weakly.

The above consideration (fig.5) corresponds to fixed parameters of standard solar model. In fact, one should consider the central temperature, $T_{c}$, abundances, $X, Y, Z$ and others as free parameters and carry out the multidimensional analysis, which includes both solar and neutrino parameters. In that time the crucial signature of resonant conversion, which enables to distinguish its effects from the astrophysical ones, is the distortion of continuous energy spectra from individual reactions (pp-, ${ }^{8} \mathrm{~B}$-decay). The distortion is described by suppression bath (fig. 3) and depends on $\Delta \mathrm{m}^{2}$ and $\sin ^{2} 2 \theta$.

Spin-flavor conversion may result in strong suppression of both ${ }^{37} \mathrm{Ar}$ production rate and number of $v$ e-scattering events as well as in the variety of the energy spectrum distortion, if the transition magnetic moment $\mu_{V}>10^{-11} \mu_{B}\left(\mu_{B}\right.$ is the Bohr magneton).

Possible anticorrelations of solar neutrino flux and 11-years solar activity [23] can be explained immediately by the magnetic field variations via the spin-flavor conversion. But it is not excluded that the change of $v$-flux is related to the variations of density distribution which influences on flavor conversion (see sect.4). 


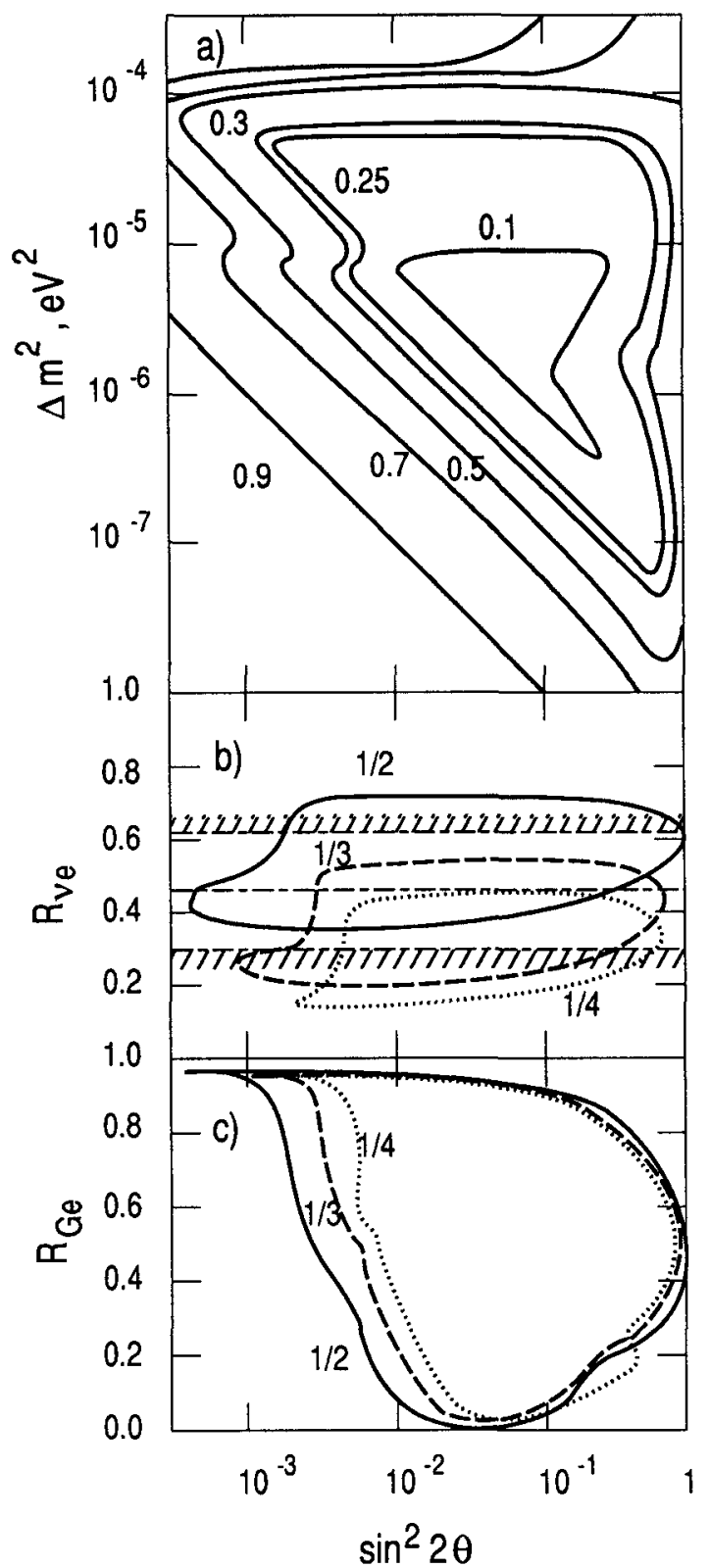

Figure 5.a). Lines of equal suppression (ISOSNU lines) of $37_{A r}$ production rate in CI-Ar experiment. Numbers on the curves are the suppression factors, $R_{A r}$.

b). The suppression factor of number of $v e-v$ e events with the energies of final electrons $E_{e}>9.5 \mathrm{MeV}$ as the function of $\sin ^{2} 2 \theta$ at fixed suppression of Ar-production rate (numbers on the curves). The points of loops correspond to definite points of ISOSNU lines. The horizontal lines are the Kamiokande-II result with $1 \sigma$ errors.

c). The same as in fig. b) for the Ge-production rate in Ga-Ge experiment. 


\section{Matter density perturbations and resonance conversion.}

The influence of density perturbations on conversion depends on relation between the oscillation length, $I_{m}$, and the spatial scale of perturbation, $I_{f I}$ (several model calculations were performed in $[28,29$, 11] ).

The smooth variations of density profile with typical scale $I_{f I} \cong R_{0}$ result in rather small change of suppression baths, which is controled by the energies $E_{c} \sim 1 / \rho$ and $E_{a} \sim d \log \rho / d r(17,18)$. Such a change reduces mainly to snift of the edges of baths and is equivalent to renormalization of $\Delta \mathrm{m}^{2}$ and $\sin ^{2} 2 \theta$. Futher on we will concentrate on the case $\mathrm{I}_{\mathrm{fI}} \cong \mathrm{I}_{\mathrm{m}}<<$ $\mathrm{R}_{\mathrm{o}}$.

\subsection{CONDITIONS FOR MAXIMAL EFFECT OF DENSITY PERTURBATIONS}

As the scales, $I_{f I}$, and amplitudes $A_{\rho}=\Delta \rho / \rho$ of possible density perturbations in the $S u n$ are essentialy unknown and moreover $A_{\rho}$ are considered to be small (no more than several percents), it is worthwhile to evaluate the minimal values of $A_{\rho}$ and $I_{f I}$ which can be observed with solar neutrino data. We begin with conditions under which the influence of $\rho$-perturbation on resonance conversion is maximal.

1). The perturbations should be placed in the resonance layer (at $\rho=$ $\left.\rho_{R}(E)\right)$. Indeed, density fluctuation, $\Delta \rho_{\mathrm{fI}}$, can be considered as the perturbation of potential : $\Delta \mathrm{V}=\sqrt{ } 2 . \mathrm{G}_{\mathrm{F}} \cdot \Delta \rho_{\mathrm{fI}} / \mathrm{m}_{\mathrm{N}}$. The perturbation induces the transition between levels with probability $P=1$ if $\Delta V$ is of order of level splitting, $\Delta \mathrm{H}$. The splitting is minimal in resonance (fig. 1b) $\Delta \mathrm{H}_{\mathrm{R}}=\sqrt{ } 2 \mathrm{G}_{\mathrm{F}} \cdot \rho_{\mathrm{R}} \cdot \tan 2 \theta / \mathrm{m}_{\mathrm{N}}$, and from the inequality $\Delta \mathrm{V}>\Delta \mathrm{H}$ one obtains $\Delta \rho_{\mathrm{fI}}>\Delta \rho_{R}$ or $A_{\rho}=\Delta \rho_{\mathrm{fI}} / \rho>\tan 2 \theta$. The smaller $R_{\theta}$, the smaller $A_{\rho}$ is sufficient for a large effect.

2). To avoid the averaging, which suppresses the perturbation effect, neutrino should be in definite level. Maximal effect takes place in the region of nonoscillatory transformation -. on the bottom of the bath.

3 ). The effect can be additionally enhanced if there are several density fluctuations, $\mathrm{N}_{\mathrm{fI}}$, on the way of neutrinos, and the parametric condition is fulfilled $[30,31]$ :

$$
l_{f l} \cong l_{m}(\rho)
$$

In this case the effects of individual fluctuations add constructively. Now the same change of conversion probability can be obtained with smaller value of $A_{\rho}: A_{\rho} \sim 1 / N_{f I}$. If the perturbations are distributed randomly in space, then a stochastic enhancement takes place and $A \rho$ $1 / \sqrt{ } \mathrm{N}$ fr. 
4). The effect depends on the shape of density perturbation but in any cases perturbations should violate adiabaticity. Conditions 1), and 2). for a given $\rho$-fluctuations can be satisfied at definite neutrino energy only. Moreover they are compatible if the relation between the $I_{f l}$ and the position of fluctuation inside the $S$ un, $r_{0}$ is fulfilled :

$$
l_{f l}=\frac{2 \pi m_{N}}{\rho\left(r_{0}\right) \sqrt{2} G_{F} \tan 2 \theta}
$$

The effect of density fluctuation looks like a peak on the bottom of the bath (under the considered conditions). The height of the peak equals

$$
P^{\max } \approx\left(\frac{A_{\rho} N_{f l}}{2 \tan 2 \theta}\right)^{2}
$$

and the width is iversely proportional to number of structures $\Delta \mathrm{E} \sim \tan$ $2 \theta / \mathrm{N}_{\mathrm{fI}}$. If the condition (21) is not fulfilled, then two peaks appear corresponding to the resonance at $E=E_{R}$ and to parametric condition at $E=E_{P}$ (parametric resonance). If neutrino state contains the admixtures of both levels (which is realized on the nonadiabatic edge), then the $\rho$ perturbation results in that the enhancement of $P$ will be altered with energy by suppression.

\subsection{EFFECTS OF $\rho$-PERTURBATION. NUMERICAL RESULTS}

To illustrate the results discussed above Krastev and myself have calculated the probabilities of resonant conversion (averaged over oscillations in final state) for

$$
\rho(r)=\rho^{s s m}(r) \cdot\left[1+A_{\rho} \theta\left(r-r_{0}\right) \cdot \theta\left(r_{1}-r\right) \cdot \sin 2 \pi r / l_{f l}\right]
$$

where $\rho^{s s m}$ is the profile of standard solar model, $\theta(r)$ is the stepfunction (the perturbations are in the interval $r \div r_{1}$ ), $A_{\rho}=$ const. In fig. 6 the effects on the bottom of the bath $(a-c)$ and on the nonadiabatic edge (d) are shown.

\subsection{DENSITY VARIATIONS AND SOLAR NEUTRINO FLUX}

Let us adopt that variations of boron neutrinos can be observed if they take place in the energy region $\Delta E / E \geq 0.2$ and if the amplitude of variations is $\Delta P / P \geq 0.3$. For beryllium neutrinos line the region of variations can be much smaller $\Delta \mathrm{E} / \mathrm{E}>0.02$ and even very narrow peaks of fig. 6 can induce an appreciable effect. Using the results of sect. $4.2,3$. the parameters of smallest density fluctuations $\left(I_{\mathrm{fI}} \mathrm{A}_{\mathrm{p}}\right)$ are 
evaluated, which give an observable variations : $\left(\rho 10^{-3} R_{\odot}, 3 \cdot 10^{-3}\right)$ at $r_{0}$ $=0.2 R_{\odot},\left(10^{-2} R_{\ominus}, 0.03\right)$ at $r_{0}=0.5 R_{\odot}\left(0.1 R_{\ominus}, 0.1\right)$ at $r_{0}=0.7 R_{\odot}$.
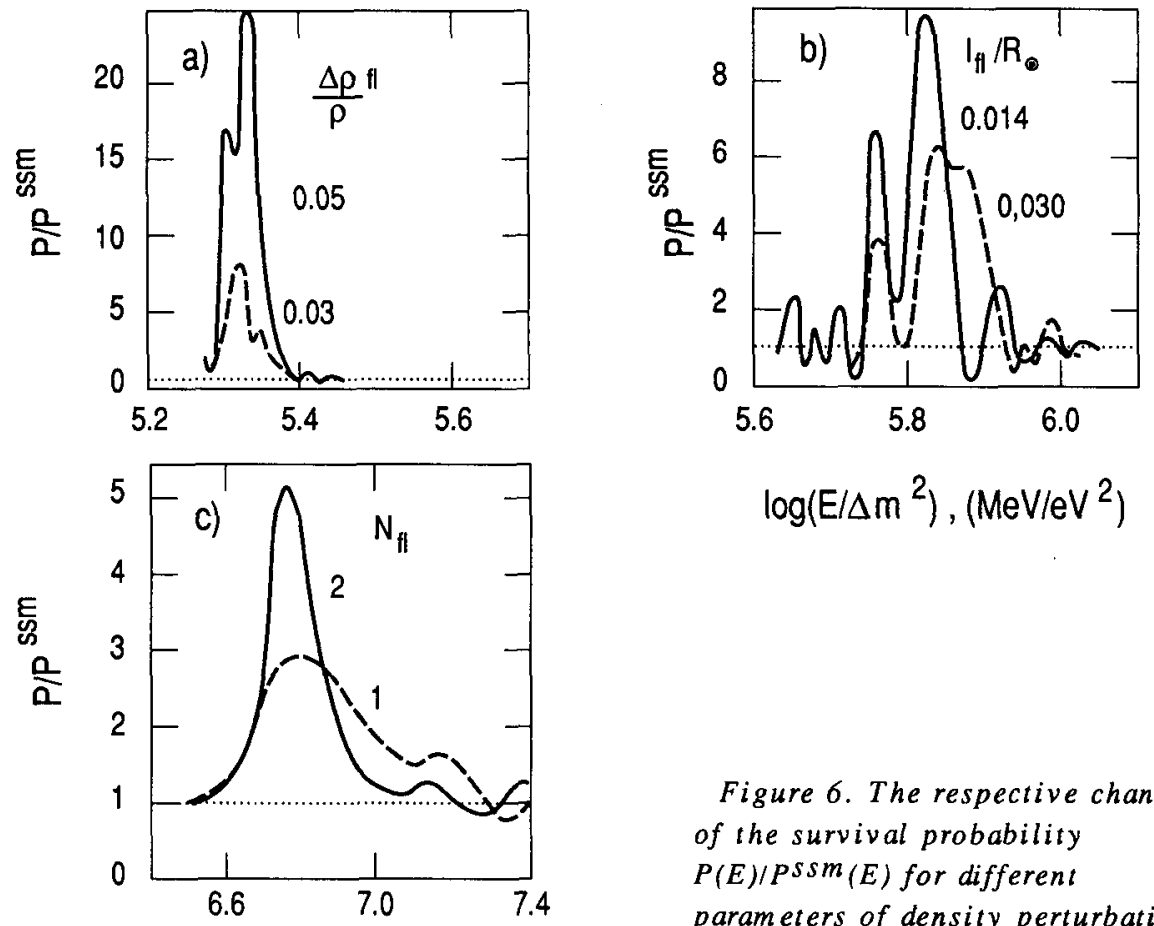

$\log \left(E / \Delta m^{2}\right),\left(M e V / e V^{2}\right)$

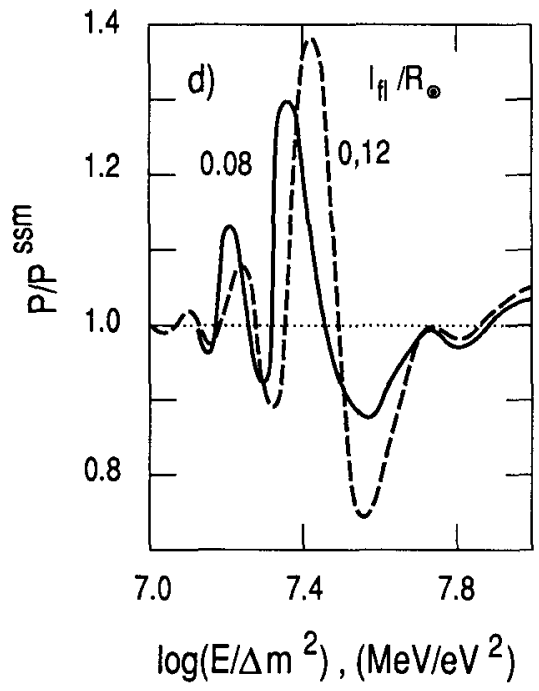

Figure 6. The respective change of the survival probability $P(E) / P s s m(E)$ for different parameters of density perturbations : $r_{0}$ - the position inside the Sun, A $\rho$ the amplitude, I $f I^{-t h e}$ spatial scale in radial direction, $N_{f I}$ - the number of periodic structures.
a). $r_{0}=0.18 R_{\odot}, \sin ^{2} 2 \theta=0.01, N_{f l}=1, I_{f I}=0 ; 015 R_{\emptyset}$.
b). $r_{0}=0.30 R_{0}, \sin ^{2} 2 \theta=0.01, \mathrm{NfI}=1, \mathrm{~A}_{\rho}=0.05$.
c). $r_{0}=0.51 R_{\odot}, \sin ^{2} 2 \theta=0.25, A_{\rho}=0.2, I_{f I}=0.035 R_{\odot}$
d). $r_{0}=0.74 R_{\rho}, \sin ^{2} 2 \theta=0.25, A_{\rho}=0.2, N_{f l}=2$. 
The most strong respective change of $P(E)$ occurs on the bottom of the baths, where in absence of $\rho$-perturbation the suppression of $v$-flux is strong. Then a scenario can be considered in which without conversion the $v$-flux is large, so that $\mathrm{Q}^{\circ} \mathrm{Ar}=(13-15) \mathrm{SNU}$ but due to flavor conversion and $\rho-v a r i a t i o n s$ the $Q_{A r}$ change from 1 to 4-5 SNU [31].

Density variations may be related to the magnetic fields [32], to the instabilities induced by rotation or nuclear energy release. Semiannual variations of signals on the Earth may be stipulated by nonsphericity of the Sun and to the annual change of Earth position. Another possibility is the $\rho$-variations due to $g$-mode of oscillations of the $S$ un; the parametric and/or stochastic enhancement of perturbation effect may take place. The change of the oscillation activity (power of vibrations) with time can induce the corresponding change of $v$-signals.

\section{Conclusion}

1). Resonant neutrino conversion -- the change of flavor of neutrino state according to matter density variations -- is the analog of well known and established phenomena from different fields of physics.

2 ). For conversion the massiveness and mixing of neutrinos are needed only. Conversion takes place inside the Sun if $\Delta \mathrm{m}^{2}=\left(10^{-8}-10^{-4}\right) \mathrm{eV}^{2}$ and $\sin ^{2} 2 \theta>10^{-4}$. Spin-flavor precession demands sufficiently large magnetic moment of neutrino: $>10^{-11} \mu_{\mathrm{B}}$.

3). Resonant conversion allows to explain the suppressions of signals in CI-Ar and Kamiokande-II experiments. Moreover it can explain the possible variations of signals via magnetic field variations (in the spinflavor case) or via density profile variations with $\Delta \rho / \rho>3.10^{-3}$ and $I_{f I}>$ $10^{-3} R_{\odot}$ (in flavor as well as spin-flavor cases). Inverseiy, the search for time variations of solar neutrino signals is sensitive to $\Delta \rho / \rho>3 \cdot 10^{-3}$ and $\mathrm{I}_{\mathrm{fI}}>10^{-3} \mathrm{R}_{\odot}$

4). Resonant conversion gives the unique method of measuring the neutrino masses and mixing in very plausible region of magnitudes, which is not achieved by usual experiments. If the conversion effects will not be found then large region of $\Delta \mathrm{m}^{2}$ and $\sin ^{2} 2 \theta$ as well as $\mu_{v}>$ $10^{-11} \mu_{\mathrm{B}}$ can be excluded.

\section{Acknowledgements}

I am gratefull to J. Boratav, M. Cribier, E. Schatzman and D. Vignaud for the discussions and hospitality during my visit in France. 


\section{References}

1. S.P. Mikheyev and A. Yu. Smirnov, Sov.J. Nucl.Phys., 42 (1985) 913-919; Nuovo Cimento, C9 (1986) 17-26.

2. S.P. Mikheyev and A. Yu. Smirnov, Sov.Phys.JETP, 64 (1986) 47.

3. L. Wolfenstein, Phys. Rev.D17 (1978) 2369-2374; ibidem D20 (1979) 2634-2635.

4. S.P. Mikheyev and A. Yu.S mirnov, Usp. Fiz. Nauk, 153 (1987) 3-58.

5. S.P. Mikheyev and A. Yu. Smirnov, Prog. in Particle and Nucl.Phys., Pergamon Press (to be published) (1989)

6. T.K. Kuo and J. Pantaleone, Rev.Mod.Phys., (1989) to be published.

7. H. Bethe, Phys.Rev.Lett., 56 (1986) 1305-1308.

8. N. Cabibbo, 10th Int. Workshop on weak interactions and neutrinos (Savonlinna, Finland 1985) unpublished.

9. A. Messiah Proc. of the 6th Moriond Workshop on Massive

Neutrinos in Astrophysics and Particle Physics (Tignes, France) eds.

O. Fackler and J. Tran Thahn Van, 373-389.

10. V. Barger et al, Phys. Rev., D34 (1986) 980-983.

11. A. Yu. Smirnov Proc. of the Int. Conf. "Neutrino-88", and Preprint MPI-PAE/PTh 48/88.

12. S.J. Parke, Phys.Rev.Lett, 57 (1986) 1275-1278.

13. W.C. Haxton, Phys.Rev.Lett., 57 (1986) 1271-1274.

14. P.I. Krastev and S.T. Petcov, Phys. Lett.B205 (1988) 84-92 and references therein.

15. S.P. Mikheyev and A. Yu. Smirnov Proc. of the 12th Int. Conf.

Neutrino-86, (Sendai, Japan), eds. T. Kitagaki and H. Yuta 177-193.

16. J. Bouches et al, Z. Phys., C32 (1986) 499-511.

V.K. Ermilova et al, Sov.JETP Letters, 43 (1986) 353-355.

17. T.K. Kuo and J. Pantaleone, Phys.Rev.Lett., 57 (1986) 1805 1808.

18. M.B. Voloshin et al, Sov.JETP, 91 (1986) 754-765, and E. Kh. Akhmedov, see in [19].

19. E. Kh. Akhmedov, Phys.Lett., 213B (1988) 64-68.

20. E. Kh. Akhmedov and O.V. Bychuk, ZhETF, 95 (1989) 442-457 
21. J.N. Bahcall and R.K. Ulrich, Rev.Mod.Phys., 60 (1988) 297372 .

22. S.J. Parke and T.P. Walker, Phys. Rev.lett., 57 (1986) 23522355 .

23. R. Davis, in "Inside the Sun" eds. G. Berthomieu and M. Cribier, Kluwer Academic Publishers, Dordrecht.

24. S.P. Mikheyev and A. Yu. Smirnov, Phys. Lett., 200 B (1988) 560564.

25. M. Nakahata in "Inside the Sun", eds. G. Berthomieu and M. Cribier, Kluwer Academic Plublishers, Dordrecht.

26. V. Gavrin in "Inside the Sun" (see ref.25).

27. T. Kirsten in "Inside the Sun" (see ref. 25).

28. A. Schafer and S.E. Koonin, Phys.Lett., B 185 (1987) 417-420.

29. M. Cribier and D. Vignaud, see in ref. [32].

30. V.K. Ermilova et al, Kr.Soob.Fiz. Lebedev Institute, 5, (1986) 26.

31. P.I. Krastev and A. Yu. Smirnov, Phys. Lett. (1989) to be published.

32. E. Schatzman and E. Ribes, Proc. of the 7 th Moriond Workshop "New and Exotic phenomena", ed. by O. Fackler and J. Tran Thanh Van, (1987) 368. 